\title{
Spherical albedo of a Lommel-Seeliger scattering ellipsoidal asteroid
}

\author{
Karri Muinonen ${ }^{1,2}$ and Olli Wilkman ${ }^{1}$ \\ ${ }^{1}$ Department of Physics, University of Helsinki, \\ Gustaf Hällströmin katu 2a, P.O. Box 64, FI-00014 U. Helsinki, Finland \\ email: karri.muinonen@helsinki.fi \\ ${ }^{2}$ Finnish Geospatial Research Institute, \\ Geodeetinrinne 2, FI-02430 Masala, Finland
}

\begin{abstract}
We compute the spherical albedo for a Lommel-Seeliger scattering ellipsoidal asteroid with a realistic disk-integrated phase function. The spherical (or Bond) albedo gives the ratio of the fluxes incident on and scattered by an asteroid. Thus, it plays a key role in the determination of the flux absorbed and afterwards thermally emitted by the asteroid at longer wavelengths. We provide extensive computations for the spherical albedo of low-albedo and moderate-albedo asteroids by utilizing the analytical disk-integrated brightness of a Lommel-Seeliger ellipsoid. In doing so, we utilize realistic triaxial models of known asteroids as well as idealistic prolate or oblate models of substantial elongation or flatness, respectively. We show that the spherical albedos can vary significantly as a function of the rotational pole orientation, rotational phase, and the triaxial ellipsoidal shape: variations of the order of 5-10\% are realistic, with a tendency to grow with increasing elongation or flatness of the shape.
\end{abstract}

Keywords. Minor planets, asteroids, solar system: general, radiative transfer, scattering, methods: analytical, methods: numerical, techniques: photometric

\section{Introduction}

The ellipsoid captures an asteroid's shape in three dimensions with only two parameters, that is, the axial ratios $b / a$ and $c / a$, where $a, b$, and $c$ denote the semiaxes of the ellipsoid. The ellipsoid is thus an appealing overall model for an asteroid's shape, a statement supported by the scientific preparation for the Gaia space mission (Cellino et al. 2009, Carbognani et al. 2012, Cellino et al. 2015, Muinonen \& Lumme 2015, Muinonen et al. 2015) and earlier work in shape and rotational pole determination (Drummond et al. 1988, Magnusson et al. 1989).

The disk-integrated brightness of an ellipsoidal asteroid is analytically available for a Lommel-Seeliger surface scattering model, that is, in the case of dark, particulate surfaces typical for, e.g., primitive asteroids (Muinonen \& Lumme 2015). The Lommel-Seeliger model follows from the radiative-transfer theory in the case of small single-scattering albedo, allowing us to omit the orders of scattering higher than the first (Chandrasekhar 1960). The analytical disk-integrated brightness has given rise to novel initial methods for asteroid lightcurve inversion (Cellino et al. 2015, Muinonen et al. 2015) in the case of sparse photometric data.

In Section 2, we review the photometric properties of the triaxial Lommel-Seeliger ellipsoid, including the disk-integrated brightness, geometric albedo, and the spherical albedo (or Bond albedo). In Section 3, we describe extensive computations for the spherical albedo as a function of rotational pole orientation, rotational phase, as well as the ellipsoid axial ratios. Finally, in Section 4, we provide the conclusions of the study. 


\section{Photometry with a Lommel-Seeliger Ellipsoid}

We begin with the reflection coefficient $R$ of a surface element that relates the incident flux density $\pi F_{0}$ and the emergent intensity $I$ :

$$
\begin{aligned}
I\left(\mu, \phi ; \mu_{0}, \phi_{0}\right)= & \mu_{0} R\left(\mu, \phi ; \mu_{0}, \phi_{0}\right) F_{0}, \\
& \mu_{0}=\cos \iota, \mu=\cos \epsilon,
\end{aligned}
$$

where $\iota$ and $\epsilon$ are the incidence and emergence angles as measured from the outward normal vector, and $\phi_{0}$ and $\phi$ are the corresponding azimuths. We can measure $\phi$ so that the backscattering direction (or light-source direction) is with $\phi=0^{\circ}$. Thus, with the typical additional assumption of a geometrically isotropic surface, specifying $\phi_{0}$ is unnecessary.

The Lommel-Seeliger reflection coefficient (subscript LS) is (e.g., Lumme and Bowell 1981),

$$
R_{\mathrm{LS}}\left(\mu, \mu_{0}, \phi\right)=\frac{1}{4} \tilde{\omega} P(\alpha) \frac{1}{\mu+\mu_{0}},
$$

where $\tilde{\omega}$ and $P$ are the single-scattering albedo and phase function, respectively, and $\alpha$ is the phase angle, the angle between the Sun and the observer as seen from the object. The Lommel-Seeliger reflection coefficient is the first-order multiple-scattering approximation from the radiative-transfer theory (e.g., Chandrasekhar 1960). In scalar radiative transfer omitting polarization effects, the phase function $P$ provides the angular distribution of scattered light in an individual interaction and is normalized so that

$$
\int_{(4 \pi)} \frac{d \Omega}{4 \pi} P(\alpha)=1
$$

The disk-integrated brightness $L$ equals the surface integral

$$
L(\alpha)=\int_{A_{+}} d A \mu I\left(\mu, \mu_{0}, \alpha\right)=\int_{A_{+}} d A \mu \mu_{0} R\left(\mu, \mu_{0}, \alpha\right) F_{0},
$$

where $A_{+}$stands for the part of the surface that is both illuminated by the Sun and visible to the observer. For a nonspherical asteroid, $L$ can depend strongly on the orientation of the asteroid with respect to the scattering plane, where $L$ is measured.

For a spherical asteroid with diameter $D$, we obtain the disk-integrated brightness

$$
\begin{aligned}
L_{\mathrm{LS}}(\alpha) & =\frac{1}{32} \pi F_{0} D^{2} \tilde{\omega} P(\alpha) \Phi_{\mathrm{LS}}(\alpha), \\
\Phi_{\mathrm{LS}}(\alpha) & =1-\sin \frac{1}{2} \alpha \tan \frac{1}{2} \alpha \ln \left(\cot \frac{1}{4} \alpha\right),
\end{aligned}
$$

where we have also given the phase function $\Phi_{\mathrm{LS}}(\alpha)$ normalized to unity at $\alpha=0^{\circ}$.

For an ellipsoidal asteroid with the semiaxes $a, b$, and $c$, and $C=\operatorname{diag}\left(a^{-2}, b^{-2}, c^{-2}\right)$, the disk-integrated brightness is also available in a closed form. Let $\mathbf{e}_{\odot}$ and $\mathbf{e}_{\oplus}$ be the unit vectors in the directions of the Sun and the observer, respectively, as seen in the principal-axes reference frame of the ellipsoid. Following Muinonen \& Lumme (2015), for the computation of the disk-integrated brightness, we define three sets of auxiliary quantities. First, the solar phase angle follows from

$$
\cos \alpha=\mathbf{e}_{\odot} \cdot \mathbf{e}_{\oplus} \cdot
$$

Second, we define two vector-matrix products $S_{\odot}$ and $S_{\oplus}$ as well as an angle $\alpha^{\prime}$ so that

$$
S_{\odot}=\sqrt{\mathbf{e}_{\odot}^{\mathrm{T}} C \mathbf{e}_{\odot}}, S_{\oplus}=\sqrt{\mathbf{e}_{\oplus}^{\mathrm{T}} C \mathbf{e}_{\oplus}},
$$




$$
\cos \alpha^{\prime}=\frac{\mathbf{e}_{\odot}^{\mathrm{T}} C \mathbf{e}_{\oplus}}{S_{\odot} S_{\oplus}}, \sin \alpha^{\prime}=\sqrt{1-\cos ^{2} \alpha^{\prime}} .
$$

Third, we define an amplitude $S$ and an angle $\lambda^{\prime}$ so that

$$
\begin{aligned}
S= & \sqrt{S_{\odot}^{2}+S_{\oplus}^{2}+2 S_{\odot} S_{\oplus} \cos \alpha^{\prime}}, \\
& \cos \lambda^{\prime}=\frac{S_{\odot}+S_{\oplus} \cos \alpha^{\prime}}{S}, \quad \sin \lambda^{\prime}=\frac{S_{\oplus} \sin \alpha^{\prime}}{S} .
\end{aligned}
$$

The disk-integrated brightness of a Lommel-Seeliger ellipsoid is then given by

$$
\begin{gathered}
L(\alpha)=\frac{1}{8} \pi F_{0} \tilde{\omega} P(\alpha) a b c \frac{S_{\odot} S_{\oplus}}{S} . \\
\left\{\cos \left(\lambda^{\prime}-\alpha^{\prime}\right)+\cos \lambda^{\prime}+\sin \lambda^{\prime} \sin \left(\lambda^{\prime}-\alpha^{\prime}\right) \ln \left[\cot \frac{1}{2} \lambda^{\prime} \cot \frac{1}{2}\left(\alpha^{\prime}-\lambda^{\prime}\right)\right]\right\} .
\end{gathered}
$$

The geometric albedo $p$ is the disk-integrated brightness at opposition divided by that of a normally illuminated, perfectly Lambertian disk with the same surface area:

$$
p=\frac{L\left(0^{\circ}\right)}{\pi a b c S_{\odot} F_{0}}=\frac{1}{8} \tilde{\omega} P\left(0^{\circ}\right) .
$$

Notice that, for a Lommel-Seeliger ellipsoid, $p$ is constant and independent of the rotational pole orientation, rotational phase, and axial ratios.

Consider next $\tilde{\omega}$ and $P(\alpha)$. Asteroid phase curves suggest that the disk-integrated brightness phase function should be close to the $H, G_{1}, G_{2}$ phase function $\Phi_{H G_{1} G_{2}}$ (Muinonen et al. 2010). It is now reasonable to assume that the model

$$
\frac{1}{8} \tilde{\omega} P(\alpha)=p \frac{\Phi_{H G_{1} G_{2}}(\alpha)}{\Phi_{\mathrm{LS}}(\alpha)}
$$

can serve well in asteroid phase-curve analyses (Muinonen et al. 2015). Alternatively, as in the present context, we may utilize the $H, G_{12}$ phase function $\Phi_{H G_{12}}$ (Muinonen et al. 2010):

$$
\frac{1}{8} \tilde{\omega} P(\alpha)=p \frac{\Phi_{H G_{12}}(\alpha)}{\Phi_{\mathrm{LS}}(\alpha)} .
$$

Finally, the spherical albedo $A$ is obtained by integrating the disk-integrated brightness over the full solid angle (corresponding to the integration over the observing directions) and by dividing with the total power incident on the cross-sectional area of the object:

$$
\begin{aligned}
A= & \frac{1}{\pi F_{0} \pi a b c S_{\odot}} \int_{(4 \pi)} d \Omega_{\oplus} L(\alpha)=\int_{(4 \pi)} d \Omega_{\oplus} \frac{1}{8} \tilde{\omega} P(\alpha) \frac{S_{\oplus}}{S} \\
& \left\{\cos \left(\lambda^{\prime}-\alpha^{\prime}\right)+\cos \lambda^{\prime}+\sin \lambda^{\prime} \sin \left(\lambda^{\prime}-\alpha^{\prime}\right) \ln \left[\cot \frac{1}{2} \lambda^{\prime} \cot \frac{1}{2}\left(\alpha^{\prime}-\lambda^{\prime}\right)\right]\right\} \\
= & \frac{p}{\pi} \int_{(4 \pi)} d \Omega_{\oplus} \frac{\Phi_{H G_{1} G_{2}}(\alpha)}{\Phi_{\mathrm{LS}}(\alpha)} \frac{S_{\oplus}}{S} \\
& \left\{\cos \left(\lambda^{\prime}-\alpha^{\prime}\right)+\cos \lambda^{\prime}+\sin \lambda^{\prime} \sin \left(\lambda^{\prime}-\alpha^{\prime}\right) \ln \left[\cot \frac{1}{2} \lambda^{\prime} \cot \frac{1}{2}\left(\alpha^{\prime}-\lambda^{\prime}\right)\right]\right\}
\end{aligned}
$$

where, when deemed appropriate, the phase function $\Phi_{H G_{1} G_{2}}$ can be replaced with the phase function $\Phi_{H G_{12}}$. 


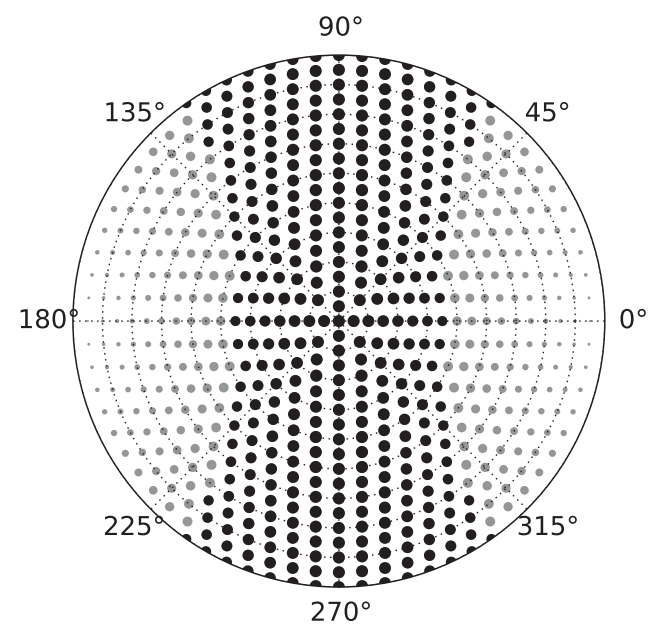

Figure 1. The pattern of average spherical albedo over one rotation as a function of the pole orientation in one sky hemisphere. The size of the dot is scaled for the $\left[A_{\min }, A_{\max }\right]$ range. The Sun is located in the $0^{\circ}$ longitude direction. The pattern is symmetric in both hemispheres. It is also practically the same for all ellipsoids, only the scaling varies. Black points indicate the range, where the albedo is larger than the mean albedo.

\section{Results and Discussion}

We compute the spherical albedo using Eq. 2.13 with the help of the Gauss-Legendre integration scheme for the cosine of the phase angle $\left(\mu_{\alpha}=\cos \alpha\right)$ and a uniform integration scheme for the aximuthal angle. It is noteworthy that, in order to arrive at accurate spherical albedos with four significant decimal places, high-resolution integration schemes are necessary for both polar and azimuthal integration: we utilize 256 and 720 integration points in $\mu_{\alpha}$ and the azimuthal angle, respectively. A typical computation for 649 pole orientations (a $5^{\circ}$ resolution across half of the full solid angle using triangulation, cf. Muinonen et al. 2015) and 24 rotational phases for each pole orientation takes about an hour of computing time using a single core on a modern computer.

We compute the spherical albedo for four different ellipsoids, using two different sets of $G_{12}$ and geometric albedo $p$ selected in accordance with the studies by Shevchenko et al. (2016), Penttilä et al. (2016), Muinonen et al. (2010), and references therein. For the darker, "C-type" models, we use $G_{12}=0.80$ and $p=0.05$. For the brighter, "S-type" models, the values are $G_{12}=0.30$ and $p=0.20$. We compare the spherical albedos of the ellipsoidal asteroids to those of a spherical asteroid with the same scattering parameters. For our C-type, the spherical asteroid has the spherical albedo $A_{\mathrm{S}}=0.018$ and, for our S-type, $A_{\mathrm{S}}=0.086$. The ellipsoid axial ratios mimic those obtained for asteroids (19) Fortuna and (1580) Betulia by Torppa et al. (2008): $b / a=0.86, c / a=0.82$ and $b / a=0.88, c / a=0.63$, respectively. In addition, we incorporate an oblate spheroid with $b / a=1.0, c / a=0.5$ and a prolate spheroid with $b / a=0.5, c / a=0.5$.

We find that the spherical albedo depends on the pole orientation and rotational aspect of an asteroid. Figure 1 shows how the ellipsoid spherical albedo $A$, averaged over the rotational phase, varies over different pole orientations. The relationship between $A$ and the pole orientation is similar for all asteroid models studied, only the relative scaling between them varies. The highest values are found when the spin axis is perpendicular to the reference plane. The values become lower towards the plane differently near the shorter and longer axis. 


\begin{tabular}{r|cccccc}
\hline Model & $b / a$ & $c / a$ & $A_{\min }$ & $A_{\max }$ & $A_{\mathrm{m}}$ & $\delta A$ \\
\hline 1C & 0.86 & 0.82 & 1.84 & 1.89 & 1.86 & 2.2 \\
2C & 0.88 & 0.63 & 1.81 & 1.91 & 1.86 & 5.1 \\
3C & 1.00 & 0.50 & 1.79 & 1.93 & 1.87 & 7.5 \\
4C & 0.50 & 0.50 & 1.83 & 1.98 & 1.87 & 8.2 \\
\hline $1 \mathrm{~S}$ & 0.86 & 0.82 & 8.53 & 8.73 & 8.61 & 2.4 \\
2S & 0.88 & 0.63 & 8.37 & 8.85 & 8.62 & 5.5 \\
3S & 1.00 & 0.50 & 8.24 & 8.94 & 8.64 & 8.1 \\
$4 \mathrm{~S}$ & 0.50 & 0.50 & 8.44 & 9.20 & 8.63 & 8.9 \\
\hline
\end{tabular}

Table 1. The ellipsoid axial ratios $b / a$ and $c / a$ and the minimum, maximum, and mean spherical albedos $A_{\min }, A_{\max }$, and $A_{\mathrm{m}}$ (all in \%) of the ellipsoid, respectively, as well as the relative variation $\delta A(\%)$, for four different shapes (1-4) and the two different spectral types (C and S). See text.
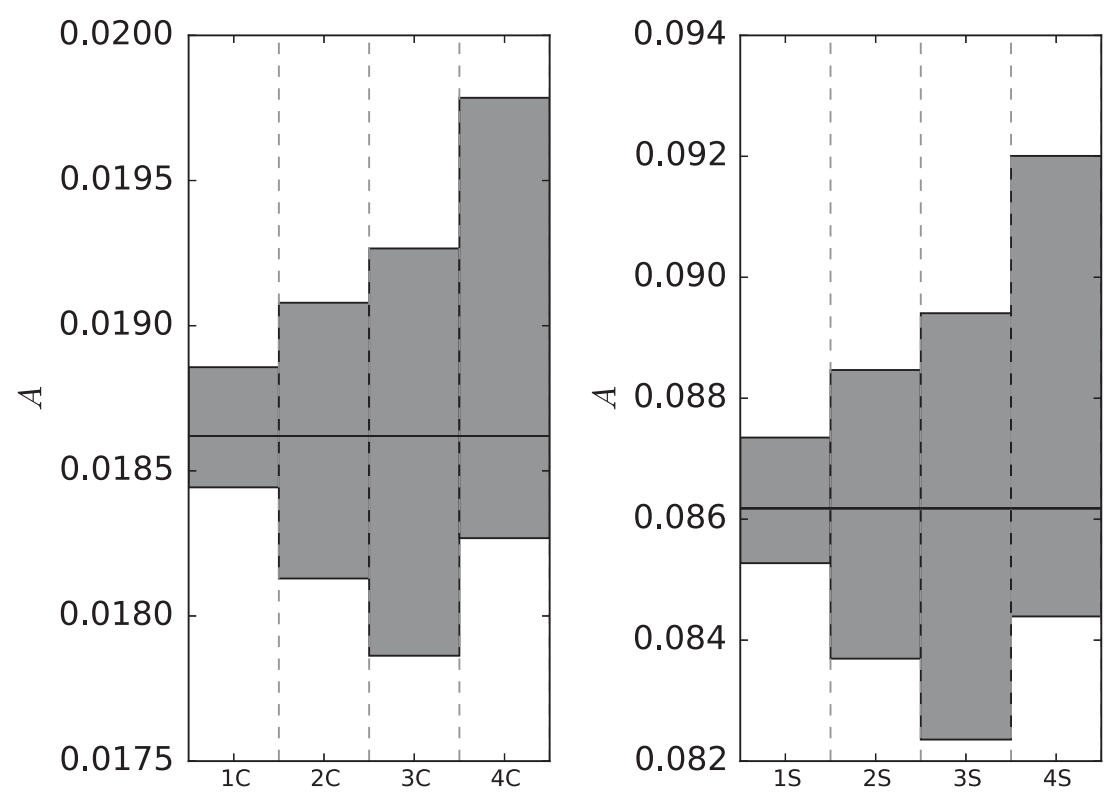

Figure 2. The range of $A$ for the different models. Grey bars show the range between the minimum and maximum values around the values for the spherical models (black lines).

Figure 2 and Table 1 show the range of variation of $A$ for each model. These values represent the maximum variation over all values computed over every pole orientation and rotational aspect. $A$ is seen to vary around the value for spheres. The relative variation of the spherical albedo,

$$
\delta A=\frac{A_{\mathrm{max}}-A_{\mathrm{min}}}{A_{\mathrm{S}}},
$$

depends mostly on the shape. For a given shape, $\delta A$ does not change much between the Cand S-types. The maximum variations of spherical albedo for a single object are almost $9 \%$. Averaging the spherical albedo over one rotation does not change the numbers of Table 1 within the precision they are given. 


\section{Conclusion}

We have computed the spherical albedo for ellipsoidal asteroids that scatter light according to the Lommel-Seeliger first-order multiple-scattering model with a realistic phase function. The results show that variations in the spherical albedo of the order of $10 \%$ can be expected.

In the future, we plan to compute reference spherical albedos for ellipsoidal asteroids as averages over all orientations weighted by the cross-sectional area of each orientation. We aim toward extending the present analysis further by incorporating the numerical particulate-medium scattering model by Wilkman et al. (2015), where the surface porosity and roughness are accounted for in detail. Extensive computations of spherical albedo are made possible by the rapid utilization of the scattering models via interpolation in a pre-computed grid of porosity and roughness parameter values.

\section{Acknowledgements}

Research supported, in part, by the Academy of Finland (grant No. 1257966).

\section{References}

Carbognani, A., Tanga, P., Cellino, A., Delbó, M., Mottola, S., \& Marchese, E. 2012, Planet. Space Sci. 73, 80, doi:10.1016/j.pss.2011.12.002

Cellino, A., Hestroffer, D., Tanga, P., Mottola, S., \& Dell'Oro, A. 2009, Astron. Astrophys. 506, 935, doi:10.1051/0004-6361/200912134

Cellino, A., Muinonen, K., Hestroffer, D., \& Carbognani, A. 2015, Planet. Space Sci., 118, 221, doi:10.1016/j.pss.2015.09.004

Chandrasekhar, S., 1960, Radiative Transfer (Dover, New York)

Drummond, J. D., Weidenshilling, S. J., Chapman, C. R., \& Davis, D. R. 1988, Icarus 76, 19, doi:10.1016/0019-1035(88)90139-X

Lumme, K. \& Bowell, E. 1981, Astron. J. 86, 1694, doi:10.1086/113054

Magnusson, P., Barucci, M. A., Drummond, J. D., Lumme, K., Ostro, S. J., Surdej, J., Taylor, R. C., \& Zappalà, V. 1989, in: R.P. Binzel, T. Gehrels, \& M. S. Matthews (eds.), Asteroids II (Univ. of Arizona Press., Tucson), p. 66

Muinonen, K., Belskaya, I. N., Cellino, A., Delbò, M., Levasseur-Regourd, A.-C., Penttilä, A., \& Tedesco, E. F. 2010, Icarus 209, 542, doi:10.1016/j.icarus.2010.04.003

Muinonen, K. \& Lumme, K. 2015, Astron. Astrophys., A23, doi:10.1051/0004-6361/201526456

Muinonen, K., Wilkman, O., Cellino, A., Wang, X., \& Wang, Y. 2015, Planet. Space Sci., 118, 227, doi:10.1016/j.pss.2015.09.005

Penttilä, A., Muinonen, K., Shevchenko, V. G., \& Wilkman, O. 2016, Planet. Space Sci., in press, doi:10.1016/j.pss.2015.08.010

Shevchenko, V. G., Belskaya, I. N., Muinonen, K., Penttilä, A., Krugly, Y. N., Velichko, F. P., Chiorny, V. G., Slyusarev, I. C., Gaftonyuk, N. M., \& Tereschenko, I. A. 2016, Planet. Space Sci., in press

Torppa, J., Hentunen, V-P., Pääkkönen, P., Kehusmaa, P., \& Muinonen, K. 2008, Icarus 198, 91, doi:10.1016/j.icarus.2008.07.014

Wilkman, O., Muinonen, K., \& Peltoniemi, J. I. 2015, Planet. Space Sci., 118, 250, doi:10.1016/j.pss.2015.06.004 